QMW-94-30

$\mathrm{R} / 94 / 33$

hep-th/9410167

October, 1994

\title{
Unity of Superstring Dualities
}

\author{
C.M. Hull \\ Physics Department, Queen Mary and Westfield College, \\ Mile End Road, London E1 4NS, U.K. \\ and \\ P. K. TOWnsEnd \\ DAMTP, University of Cambridge, \\ Silver Street, Cambridge CB3 9EW, U.K.
}

\begin{abstract}
The effective action for type II string theory compactified on a six torus is $N=8$ supergravity, which is known to have an $E_{7}$ duality symmetry. We show that this is broken by quantum effects to a discrete subgroup, $E_{7}(\mathbb{Z})$, which contains both the T-duality group $O(6,6 ; \mathbb{Z})$ and the S-duality group $S L(2 ; \mathbb{Z})$. We present evidence for the conjecture that $E_{7}(\mathbb{Z})$ is an exact 'U-duality' symmetry of type II string theory. This conjecture requires certain extreme black hole states to be identified with massive modes of the fundamental string. The gauge bosons from the Ramond-Ramond sector couple not to string excitations but to solitons. We discuss similar issues in the context of toroidal string compactifications to other dimensions, compactifications of the type II string on $K_{3} \times T^{2}$ and compactifications of eleven-dimensional supermembrane theory.
\end{abstract}




\section{Introduction}

String theory in a given background can be formulated in terms of a sum over world-sheet fields, (super-) moduli and topologies of a world-sheet sigmamodel with the background spacetime as its target space. Different backgrounds may define the same quantum string theory, however, in which case they must be identified. The transformations between equivalent backgrounds generally define a discrete group and such discrete gauge symmetries are referred to as duality symmetries of the string theory. An example is T-duality, which relates spacetime geometries possessing a compact abelian isometry group (see [1] and references therein). The simplest case arises from compactification of the string theory on a circle since a circle of radius $R$ defines the same two-dimensional quantum field theory, and hence the same string theory, as that on a circle of radius $\alpha^{\prime} / R$. Tdualities are non-perturbative in the sigma-model coupling constant $\alpha^{\prime}$ but valid

order by order in the string coupling constant $g$. Some string theories may have additional discrete symmetries which are perturbative in $\alpha^{\prime}$ but non-perturbative in $g$. An example is the conjectured S-duality of the heterotic string compactified on a six-torus $[2,3,4]$. In this paper we investigate duality symmetries of the type II string compactified to four dimensions and present evidence for a new 'U-duality' symmetry which unifies the $\mathrm{S}$ and $\mathrm{T}$ dualities and mixes sigma-model and string coupling constants.

Consider a compactified string for which the internal space is an $n$-torus with constant metric $g_{i j}$ and antisymmetric tensor $b_{i j}$. The low-energy effective field theory includes a spacetime sigma model whose target space is the moduli space $O(n, n) /[O(n) \times O(n)]$ of the torus, and the constants $g_{i j}$ and $b_{i j}$ are the expectation values of the $n^{2}$ scalar fields. There is a natural action of $O(n, n)$ on the moduli space. In general this takes one string theory into a different one but a discrete $O(n, n ; \mathbb{Z})$ subgroup takes a given string theory into an equivalent one. This is the T-duality group of the toroidally-compactified string and the true moduli space of the string theory is the moduli space of the torus factored by the discrete T-duality 
group. There is a generalization to Narain compactifications on the '(p,q)-torus' $T(p, q)$ for which the left-moving modes of the string are compactified on a $p$ torus and the right-moving ones on a $q$-torus [5]. In this case the moduli space is $O(p, q) /[O(p) \times O(q)]$ factored by the T-duality group $O(p, q ; \mathbb{Z})$. The $T(6,22)$ case is relevant to the heterotic string compactified to four dimensions which has $O(6,22 ; \mathbb{Z})$ as its T-duality group. At a generic point in the moduli space the effective field theory is $N=4$ supergravity coupled to 22 abelian vector multiplets, giving a total of 28 abelian vector gauge fields [6] with gauge group $U(1)^{28}$. It follows from the compactness of the full gauge group for all 28 vector gauge fields that any electric or magnetic charges are quantized. The effective field theory has an $S L(2 ; \mathbb{R}) \times O(6,22)$ invariance of the equations of motion which, due to the charge quantization and the fact that states carrying all types of charge can be found in the spectrum, is broken to the discrete subgroup $S L(2 ; \mathbb{Z}) \times O(6,22 ; \mathbb{Z})$. The $O(6,22 ; \mathbb{Z})$ factor extends to the T-duality group of the full string theory. It has been conjectured that the $S L(2 ; \mathbb{Z})$ factor also extends to a symmetry of the full string theory [3]. This is the S-duality group of the heterotic string. It acts on the dilaton field $\Phi$ and the axion field $\psi$ (obtained by dualizing the four-dimensional two-form gauge field $b_{\mu \nu}$ that couples to the string) via fractional linear transformations of the complex scalar $\psi+i e^{-\Phi}$ and on the abelian field strengths by a generalized electric-magnetic duality. One of the $S L(2 ; \mathbb{Z})$ transformations interchanges the electric and magnetic fields and, when $\psi=0$, takes $\Phi$ to $-\Phi$ which, since the expectation value of $e^{\Phi}$ can be identified with the string coupling constant $g$, takes $g$ to $1 / g$, and so interchanges strong and weak coupling.

Consider now the compactification of the type IIA or type IIB superstring to four dimensions on a six-torus. The low-energy effective field theory is $N=8$ supergravity [7], which has 28 abelian vector gauge fields and 70 scalar fields taking values in $E_{7(7)} /\left[S U(8) / Z_{2}\right]$. The equations of motion are invariant under the action of $E_{7(7)}$ [7], which contains $S L(2 ; \mathbb{R}) \times O(6,6)$ as a maximal subgroup. We shall show that certain quantum mechanical effects break $E_{7(7)}$ to a discrete subgroup which we shall call $E_{7}(\mathbb{Z})$, and this implies a breaking of the maximal 
$S L(2 ; \mathbb{R}) \times O(6,6)$ subgroup to $S L(2 ; \mathbb{Z}) \times O(6,6 ; \mathbb{Z})$. The $O(6,6 ; \mathbb{Z})$ factor extends to the full string theory as the T-duality group and it is natural to conjecture that the $S L(2 ; \mathbb{Z})$ factor also extends to the full string theory as an S-duality group. In fact, we shall present evidence for the much stronger conjecture that the full $E_{7}(\mathbb{Z})$ group (to be defined below) extends to the full string theory as a new unified duality group, which we call U-duality. U-duality acts on the abelian gauge fields through a generalized electromagnetic duality and on the 70 scalar fields, the constant parts of which can each be thought of as a coupling constant of the theory. The zero-mode of the dilaton is related to the string coupling $g$, while 21 of the scalar zero-modes are the moduli of the metric on the 6-torus, and the others parameterise the space of constant anti-symmetric tensor gauge fields on the six-torus. U-duality implies that all 70 coupling constants are on a similar footing despite the fact that the standard perturbative formulation of string theory assigns a special rôle to one of them.

Whereas T-duality is known to be an exact symmetry of string theory at each order in the string coupling constant $g$, the conjectured S-duality and U-duality are non-perturbative and so cannot be established within a perturbative formulation of string theory. However, it was pointed out in [4] in the context of the heterotic string that there are a number of quantities for which the tree level results are known to be, or believed to be, exact, allowing a check on S-duality by a perturbative, or semi-classical, calculation. We shall show that U-duality for the type II string passes the same tests.

First, for compactifications of the type II string that preserve at least $N=4$ supersymmetry, the low energy effective field theory for the massless modes is a supergravity theory whose form is determined uniquely by its local symmetries and is therefore not changed by quantum corrections. Duality of the string theory therefore implies the duality invariance of the equations of motion of the supergravity theory. This prediction is easily checked because the symmetries of the $N \geq 4$ supergravity/matter theories have been known for some time. In particular, the equations of motion of $N=8$ supergravity are U-duality invariant. Another quan- 
tity that should be, and is, duality invariant is the set of values of electric and magnetic charges allowed by the Dirac-Schwinger-Zwanziger quantization condition. Third, the masses of states carrying electric or magnetic charges satisfy a Bogomolnyi bound which, for the compactifications considered here, is believed to be unrenormalized to arbitrary order in the string coupling constant. Duality invariance of the string theory requires this bound to be duality invariant. For soliton states the Bogomolnyi bound can be found from a classical bound on field configurations of the effective supergravity theory carrying electric or magnetic charges that generalizes the bound obtained in [8] for Maxwell-Einstein theory. We present this bound for $N=8$ supergravity and show that it is U-duality invariant. Fourth, the spectrum of 'Bogomolnyi states' saturating the Bogomolnyi bound should also be duality invariant. These states include winding and momentum modes of the fundamental string and those found from quantization of solitons. We shall assume that soliton solutions of the type II string can be identified with those of its effective $N=8$ supergravity theory, and these, as we shall see, include various types of extreme black hole*.

One of the main concerns of this paper will be the Bogomolnyi states of the type II string theory that break half the supersymmetry. As we shall see, the soliton states in this category arise from quantization of a particular class of extreme black hole solution of $N=8$ supergravity. It is essentially automatic that all soliton states of the type II string fall into representations of the U-duality group because this is a symmetry of the equations of motion of which the solitons are solutions $^{\dagger}$. A similar argument can be made for solitons of the heterotic string; for example, extreme black hole solutions of the low-energy field theory corresponding to the heterotic string fit into $S L(2, \mathbb{Z})$ representations [10]. There are two points to bear in mind, however. First, a duality transformation not only produces new soliton solutions from old but also changes the vacuum, as the vacuum is parame-

\footnotetext{
$\star$ See [9] for a discussion of the interpretation of extreme black holes as solitons

$\dagger$ They also fall into supermultiplets because of the fermion zero modes in the presence of an extreme black hole [11]
} 
terised by the scalar expectation values and these change under duality. We shall assume, as in [4], that the new soliton state in the new vacuum can be continued back to give a new soliton state in the old vacuum with duality transformed electric and magnetic charges; this is certainly possible at the level of solutions of the low energy effective action, since the extreme black hole solutions depend analytically on the scalar expectation values. Combining U-duality transformations with analytic continuations of the scalar field zero-modes in this way gives an $E_{7}(\mathbb{Z})$ invariance of the spectrum of soliton states in a given vacuum. (Note that whereas U-duality preserves masses, combining this with a scalar zero-mode continuation gives a transformation which changes masses and so is not an invariance of the Hamiltonian.) Second, the four-dimensional metrics of many extreme black hole solitons are only defined up to a conformal rescaling by the exponential of a scalar field function that vanishes at spatial infinity. While the 'Einstein' metric is duality invariant, other metrics in the same conformal equivalence class will not be. In general one should therefore think of duality as acting on conformal equivalence classes of metrics and the issue arises as to which metric within this class is the physically relevant one. As we shall see, for the solutions considered here each conformal class of metrics contains one that is (i) either completely regular or regular outside and on an event horizon and (ii) such that its spatial sections interpolate between topologically distinct vacua. The extreme black hole solutions corresponding to these metrics might reasonably be interpreted as solitons of the theory.

We now encounter an apparent contradiction with U-duality, and with Sduality, of the type II string theory because the fundamental string excitations include additional Bogomolnyi states which apparently cannot be assigned to duality multiplets containing solitons because the soliton multiplets are already complete. The only escape from this contradiction is to make the hypothesis that the fundamental string states have already been counted among the soliton states. In order for this to be possible there must be soliton states carrying exactly the same quantum numbers as the fundamental Bogomolnyi states. This is indeed 
the case. The idea that particles with masses larger than the Planck mass, and hence a Compton wavelength less than their Schwarzschild radius, should be regarded as black holes is an old one [12,13], and it has recently been argued that Bogomolnyi states in the excitation spectrum of the heterotic string should be identified with extreme electrically-charged dilaton black holes [14,15]. For the heterotic string, approximate solutions of the low-energy effective action include extreme black holes and self-gravitating BPS monopoles [16,17], and it is believed that these correspond to Bogomolnyi solitons of the heterotic string [4]. Any magnetically charged soliton will have an electrically charged soliton partner generated by the action of the $\mathbb{Z}_{2}$ electromagnetic duality subgroup of S-duality. Now, if the full string theory is S-duality invariant, and this $\mathbb{Z}_{2}$ subgroup acts on an electrically charged fundamental string state to give a magnetically charged soliton, as argued for the heterotic string in [4], then this fundamental string state must be identified with the corresponding electrically charged soliton. We shall return to these points later but it is worth noting here that solitons of the low-energy effective $N=4$ or $N=8$ supergravity theory fit into representations of the $S \times T$ or $U$ duality as these are symmetries of the supergravity equations of motion, and this is true irrespective of whether the duality symmetry is actually a symmetry of the full heterotic or type II string theory.

For compactifications of ten-dimensional string theories one expects solitons of the effective four-dimensional theory to have a ten-dimensional origin. For the type II string we are able to identify the four dimensional solitons that break half the supersymmetry of $N=8$ supergravity as six-torus 'compactifications' of the extreme black $p$-branes of either IIA or IIB ten-dimensional supergravity $[18,19,20,21,22]$. We note that, in this context, the Bogomolnyi bound satisfied by these states can be seen to arise from the algebra of Noether charges of the effective world-volume action [23]. Remarkably, the solitonic states that are required to be identified with fundamental string states are precisely those which have their tendimensional origin in the string soliton or extreme black 1-brane solution, which couples to the same two-form gauge field as the fundamental string. This suggests 
that we should identify the fundamental ten dimensional string with the solitonic string. This is consistent with a suggestion made in [24], for other reasons, that the four-dimensional heterotic string be identified with an axion string.

A similar analysis can be carried out for non-toroidal compactification. A particularly interesting example is compactification of the type II superstring on $K_{3} \times T^{2}$ [25] for which the effective four-dimensional field theory turns out to be identical to the effective field theory of the $T^{6}$-compactified heterotic string, and in particular has the same $S L(2, \mathbb{Z}) \times O(6,22 ; \mathbb{Z})$ duality group. Furthermore, the spectrum of extreme black hole states is also the same. This raises the possibility that the two string theories might be non-perturbatively equivalent, even though they differ perturbatively. Such an equivalence would clearly have significant implications.

Finally we consider similar issues in the context of the 11-dimensional supermembrane [26]. This couples naturally to 11-dimensional supergravity [27] and hence to $N=8$ supergravity after compactification on $T^{7}$ and to $N=4$ supergravity coupled to 22 vector multiplets after compactification on $K_{3} \times T^{3}$ [28]. At present it is not known how to make sense of a quantum supermembrane, so there is little understanding of what the massive excitations might be. However, some progress can be made using the methods sketched above for the string. We shall show that, if the elementary supermembrane is identified with the solitonic membrane solution [29] of 11-dimensional supergravity and account is taken of the solitonic fivebrane solution [30], the results of this analysis for the four-dimensional theory are exactly the same as those of the type II string. 


\section{Charge Quantization and the Bogomolnyi Bound}

Consider the four-dimensional Lagrangian

$$
L=\sqrt{-g}\left(\frac{1}{4} R-\frac{1}{2} g_{i j}(\phi) \partial_{\mu} \phi^{i} \partial^{\mu} \phi^{j}-\frac{1}{4} m_{I J}(\phi) F^{\mu \nu} F_{\mu \nu}^{J}-\frac{1}{8} \varepsilon^{\mu \nu \rho \sigma} a_{I J}(\phi) F_{\mu \nu}^{I} F_{\rho \sigma}^{J}\right)
$$

for space-time 4-metric $g_{\mu \nu}$, scalars $\phi^{i}$ taking values in a sigma-model target space $\mathcal{M}$ with metric $g_{i j}(\phi)$, and $k$ abelian vector fields $A_{\mu}^{I}$ with field strengths $F_{\mu \nu}^{I}$. The scalar functions $m_{I J}+i a_{I J}$ are entries of a positive definite $k \times k$ Hermitian matrix. The bosonic sector of all supergravity theories without scalar potentials or non-abelian gauge fields can be put in this form. We shall be interested in those cases for which the equations of motion are invariant under some symmetry group $G$, which is necessarily a subgroup of $S p(2 k ; \mathbb{R})[31]$ and an isometry group of $\mathcal{M}$. Of principal interest here are the special cases for which $\mathcal{M}$ is the homogeneous space $G / H$ where $H$ is the maximal compact subgroup of $G$. These cases include many supergravity theories, and all those with $N \geq 4$ supersymmetry. For $N=4$ supergravity coupled to $m$ vector multiplets $k=6+m, G=S L(2 ; \mathbb{R}) \times O(6, m)$ and $H=U(1) \times O(6) \times O(m)$. For $N=8$ supergravity $k=28, G=E_{7(7)}$ and $H=S U(8)$. For the 'exceptional' $N=2$ supergravity [32], $k=28, G=E_{7(-25)}$ and $H=E_{6} \times U(1)$.

Defining

$$
G_{\mu \nu I}=m_{I J} \star F_{\mu \nu}^{J}+a_{I J} F_{\mu \nu}^{J}
$$

where $\star F_{\mu \nu}^{I}=\frac{1}{2} \varepsilon_{\mu \nu \rho \sigma} F^{\rho \sigma I}$, the $A_{\mu}^{I}$ field equations and Bianchi identities can be written in terms of the the $2 k$-vector of two-forms

$$
\mathcal{F}=\left(\begin{array}{l}
F^{I} \\
G_{I}
\end{array}\right)
$$

as simply $d \mathcal{F}=0$. The group $G$ acts on the scalars through isometries of $\mathcal{M}$ and on $\mathcal{F}$ as $\mathcal{F} \rightarrow \Lambda \mathcal{F}$ where $\Lambda \in G \subseteq S p(2 k ; \mathbb{R})$ is a $2 k \times 2 k$ matrix preserving the 
$2 k \times 2 k$ matrix

$$
\Omega=\left(\begin{array}{cc}
0 & \mathbb{1} \\
-\mathbb{1} & 0
\end{array}\right)
$$

An alternative way to represent the $G / H$ sigma model is in terms of a $G$-valued field $V(x)$ which transforms under rigid $G$-transformations by right multiplication and under local $H$ transformations by left multiplication

$$
V(x) \rightarrow h(x) V(x) \Lambda^{-1} \quad h \in H, \Lambda \in G
$$

The local $H$-invariance can be used to set $V \in G / H$. Note that $\overline{\mathcal{F}} \equiv V \mathcal{F}$ is $G$ invariant. In most cases of interest, the scalar coset space can be parameterised by the complex scalars $z_{I J}=a_{I J}+i m_{I J}$ which take values in a generalised upperhalf-plane $\left(m_{I J}\right.$ is positive definite) and the group $G$ acts on $z_{I J}$ by fractional linear transformations. (This can be seen for $N=8$ supergravity as follows. In the symmetric gauge [7], the coset is parameterised by a scalar $y_{I J}$ which transforms under fractional linear transformations under $G$. However, $z_{I J}$ is related to $y_{I J}$ by a fractional linear transformation, $z_{I J}=i(\mathbb{1}+\bar{y}) /(\mathbb{1}-\bar{y})$, so that $z$ in turn transforms under $G$ by fractional linear transformations. Similar results follow for $N<8$ supergravities by truncation.)

We now define the charges

$$
Q^{I}=\oint_{\Sigma} \star F^{I} \quad p_{I}=\frac{1}{2 \pi} \oint_{\Sigma} F^{I} \quad q_{I}=\oint_{\Sigma} G_{I}
$$

as integrals of two-forms over a two-sphere $\Sigma$ at spatial infinity. The charges $p^{I}$ and $q_{I}$ are the magnetic charges and the Noether electric charges, respectively. The charges $Q^{I}$ are the electric charges describing the $1 / r^{2}$ fall-off of the radial components of the electric fields, $F_{0 r}^{I}$, and incorporate the shift in the electric charge of a dyon due to non-zero expectation values of axion fields [33]. Indeed, if 
the scalars $\phi^{i}$ tend to constant values $\bar{\phi}^{i}$ at spatial infinity, then

$$
q_{I}=m_{I J}(\bar{\phi}) Q^{J}+a_{I J}(\bar{\phi}) p^{J}
$$

The charges $\left(p^{I}, q_{I}\right)$ can be combined into a $2 k$-vector

$$
\mathcal{Z}=\oint_{\Sigma} \mathcal{F}=\left(\begin{array}{l}
p^{I} \\
q_{I}
\end{array}\right)
$$

from which it is clear that $\mathcal{Z} \rightarrow \Lambda \mathcal{Z}$ under $G$.

The Dirac-Schwinger-Zwanziger (DSZ) quantization condition (with $\hbar=1$ ) for two dyons with charge vectors $\mathcal{Z}$ and $\mathcal{Z}^{\prime}$ is

$$
\mathcal{Z}^{T} \Omega \mathcal{Z}^{\prime} \equiv p^{I} q_{I}^{\prime}-p^{\prime I} q_{I}=\nu
$$

for some integer $\nu$. This quantization condition is manifestly $G$-invariant as $G \subseteq$ $S p(2 k ; \mathbb{R})$. However, it has implications for the quantum theory only if there exist both electric and magnetic charges. If, for example, there are no magnetic charges of one type then (2.9) places no constraint on the values of the corresponding electric charge. For the cases of interest to us here, we will show that there exist electric and magnetic charges of all types. We shall now proceed with our analysis of the general case assuming all types of charge exist and this, together with the quantization condition (2.9), implies that the Noether electric charges $q_{I}$ lie in some lattice $\Gamma$ and that the magnetic charges $p^{I}$ lie in the dual lattice $\tilde{\Gamma}$. The group $G$ is therefore broken to the discrete sub-group $G(\mathbb{Z})$ which has the property that a vector $\mathcal{Z} \in \Gamma \oplus \tilde{\Gamma}$ is taken to another vector in the same self-dual lattice. The subgroup of $S p(2 k)$ preserving the lattice is $S p(2 k ; \mathbb{Z})$, so that the duality group is

$$
G(\mathbb{Z})=G \cap S p(2 k ; \mathbb{Z})
$$

For compact $G, G(\mathbb{Z})$ is a finite group while for non-compact $G$ it is an infinite discrete group. If we choose a basis for the fields $A^{I}$ so that the electric charges, 
and hence the magnetic charges, are integers, then the lattice $\Gamma \oplus \tilde{\Gamma}$ is preserved by integer-valued matrices, so that $S p(2 k ; \mathbb{Z})$ consists of integer-valued $2 k \times 2 k$ matrices preserving $\Omega$, and $G(\mathbb{Z})$ is also represented by integer-valued $2 k \times 2 k$ matrices. Note that the group $G(\mathbb{Z})$ is independent of the geometry of the lattice, as any two lattices $\Gamma, \Gamma^{\prime}$ are related by a $G L(k, \mathbb{R})$ transformation, so that the corresponding discrete groups $G(\mathbb{Z}), G^{\prime}(\mathbb{Z})$ are related by $G L(k, \mathbb{R})$ conjugation and so are isomorphic. For $N=4$ supergravity coupled to 22 vector multiplets, $G(\mathbb{Z})$ is precisely the $S \times T$ duality group $S L(2 ; \mathbb{Z}) \times O(6,22 ; \mathbb{Z})$ of the toroidallycompactified heterotic string, which was observed previously to be the quantum symmetry group of this effective field theory [4]. For $N=8$ supergravity $G(\mathbb{Z})$ is a discrete subgroup of $E_{7(7)}$ which we shall call $E_{7(7)}(\mathbb{Z})$ and abbreviate to $E_{7}(\mathbb{Z})$. It can be alternatively characterized as the subgroup of $S p(56 ; \mathbb{Z})$ preserving the invariant quartic form of $E_{7(7)}$. From the explicit form of this invariant given in [7], it is straightforward to see that $E_{7}(\mathbb{Z})$ contains an $S L(8, \mathbb{Z})$ subgroup. We also have

$$
E_{7}(\mathbb{Z}) \supset S L(2 ; \mathbb{Z}) \times O(6,6 ; \mathbb{Z})
$$

so that $E_{7}(\mathbb{Z})$ contains the T-duality group of the toroidally-compactified type II string. The minimal extension of the S-duality conjecture for the heterotic string would be to suppose that the $S L(2 ; \mathbb{Z})$ factor extends to an S-duality group of the type II string, but it is natural to conjecture that the full discrete symmetry group is the much larger U-duality group $E_{7}(\mathbb{Z}) . \quad E_{7}(\mathbb{Z})$ is strictly larger than $S L(2 ; \mathbb{Z}) \times O(6,6 ; \mathbb{Z})$, as it also contains an $S L(8, \mathbb{Z})$ subgroup. In the next section we shall verify some predictions of U-duality for the spectrum of states saturating a gravitational version of the Bogomolnyi bound, i.e. the 'Bogomolnyi states'. However, before turning to the spectrum we should verify that the Bogomolnyi bound is itself U-duality invariant, since otherwise a U-duality transformation could take a state in the Bogomolnyi spectrum to one that is not in this spectrum.

Consider first the cases of pure $N=4$ supergravity (without matter coupling) and $N=8$ supergravity, for which $\mathcal{M}=G / H$ and $k=N(N-1) / 2$. We define 
$Y_{m n}=t_{m n}^{I}\left(\bar{q}_{I}+i \bar{p}^{I}\right)$ where $\left(\bar{p}^{I}, \bar{q}_{I}\right)$ are the components of the $2 k$-vector $\overline{\mathcal{Z}}=\bar{V} \mathcal{Z}$ and $\bar{V}$ is the constant asymptotic value of the $G$-valued field $V$ at spatial infinity. Here $m, n=1, \ldots, N$ and $t_{m n}^{I}=-t_{n m}^{I}$ are the matrices generating the vector representation of $S O(N)$. The $Y_{m n}$ appear in a global supersymmetry algebra as central charges $[34,8,9,35]$ and this allows a derivation of a Bogomolnyi bound. The anti-symmetric complex $N \times N$ matrix $Y_{m n}$ has $N / 2$ complex skew eigen-values $\lambda_{a}$, $a=1, \ldots, N / 2$ and the bound on the ADM mass of the Maxwell-Einstein theory [8] can be generalized to [35]

$$
M_{A D M} \geq \max \left|\lambda_{a}\right|
$$

Since $\mathcal{Z} \rightarrow \Lambda \mathcal{Z}$ and $\bar{V} \rightarrow \bar{V} \Lambda^{-1}$ under $G$, it follows that $\overline{\mathcal{Z}}$ and the $\lambda_{a}$ are invariant under duality transformations, so the bound (2.12) is manifestly $G$-invariant. In the quantum theory this bound translates to a bound on the mass of the corresponding quantum state. Similar results apply to the case of $N=4$ super-matter coupled to supergravity, with the difference that $t_{m n}^{I}=-t_{n m}^{I}$ are now certain scalar-field dependent matrices that 'convert' the $S O(6, m)$ index $I$ to the $S O(6)$ composite index $m n$. Nevertheless, the charges $\lambda_{a}$ remain duality-invariant.

If the moduli of all the eigenvalues are equal, $\left|\lambda_{a_{1}}\right|=\left|\lambda_{a_{2}}\right|=\ldots=\left|\lambda_{a_{N / 2}}\right|$, then the bound $(2.12)$ is equivalent to

$$
M_{A D M} \geq \sqrt{\frac{2}{N}} \sqrt{|\overline{\mathcal{Z}}|^{2}}
$$

where

$$
|\overline{\mathcal{Z}}|^{2}=\sum_{a}\left|\lambda_{a}\right|^{2}=\frac{1}{2} \bar{Y}_{m n} \bar{Y}^{m n}=G_{I J} \bar{p}^{I} \bar{p}^{J}+G_{I J} \bar{q}_{I} \bar{q}_{J}
$$

and $G_{I J}=\frac{1}{2} t_{m n}^{I} t_{m n}^{J}$ is the identity matrix for pure supergravity, but is scalar dependent for the the matter-coupled $N=4$ theory. However, in the general case of different eigenvalues, the bound (2.14) is strictly weaker than (2.12). If $M_{A D M}$ is equal to the modulus of $r$ of the eigenvalues $\lambda_{a}, M_{A D M}=\left|\lambda_{a_{1}}\right|=\left|\lambda_{a_{2}}\right|=$ 
$\ldots=\left|\lambda_{a_{r}}\right|$, for some $r$ with $0 \leq r \leq N / 2$, then the soliton with these charges spontaneously breaks the $N$ original supersymmetries down to $r$ supersymmetries, so that for solitons for which $r=N / 2$ precisely half of the $N$ supersymmetries are preserved and the bound (2.12) is equivalent to (2.14). The duality invariance of the bound (2.14) for $N=4$ was previously pointed out in [3,4].

\section{Spectrum of Bogomolnyi states}

There are many massive states in the spectrum of toroidally-compactified string theories. The masses of those which do not couple to any of the $U(1)$ gauge fields cannot be calculated exactly. This is also true in general of those that do couple to one of the $U(1)$ gauge fields but the masses of such particles are bounded by their charges, as just described. It is believed that the masses of string states that saturate the bound are not renormalized for theories with at least $N=4$ supersymmetry. If this is so then these masses can be computed exactly. Such 'Bogomolnyi states' arise in the theory from winding and Kaluza-Klein modes of the fundamental string, and from quantization of non-perturbative soliton solutions of the string theory. The latter include extreme black holes and, for the heterotic string, self-gravitating BPS monopoles.

For generic compactifications of both heterotic and type II strings there are 28 abelian gauge fields and so a possible 56 types of electric or magnetic charge. We shall identify solitons of the effective supergravity theory carrying each type of charge, thereby justifying the quantization condition on these charges. These solitons are various types of extreme black holes. Initially, at least, we shall be interested in solitons carrying only one type of charge, in which case we should consistently truncate the supergravity theory to one with only one non-zero field strength, $F$. The coefficients of the $F^{2}$ terms can then be expressed in terms of a scalar field $\sigma$ and a pseudoscalar field $\rho$ (which are two functions of the $\phi^{i}$ ) such that the truncated field theory has an action of the form

$$
S=\int d^{4} x \sqrt{-g}\left(\frac{1}{4} R+\frac{1}{4} e^{-2 a \sigma} F_{\mu \nu} F^{\mu \nu}+\frac{1}{4} \rho F_{\mu \nu} \star F^{\mu \nu}+L(\sigma, \rho)\right)
$$


where $L(\sigma, \rho)$ is the lagrangian for a scalar sigma-model and $a$ is a constant. One can choose $a \geq 0$ without loss of generality since $a$ is changed to $-a$ by the field redefinition $\sigma \rightarrow-\sigma$. For every value of $a$ the equations of motion of (3.1) admit extreme multi-black hole solutions [36], parameterised by the asymptotic values of $\sigma, \rho$, which are arbitrary integration constants. There is an intrinsic ambiguity in the metric of the $a \neq 0$ extreme black hole solutions because a new metric can be constructed from the canonical metric (appearing in the action (3.1)) by a conformal rescaling by a power of $e^{\sigma}$. The general metric in this conformal equivalence class will not have an interpretation as a 'soliton' in the sense for which the $a=0$ extreme Reissner-Nordstrom (RN) black hole is a soliton. One feature that is generally expected from a soliton is that it interpolate between different vacua: in the RN case these are the Minkowski spacetime near spatial infinity and the Robinson-Bertotti vacuum down an infinite Einstein-Rosen 'throat'. If we require of the $a \neq 0$ extreme black holes that they have a similar property then one must rescale the canonical metric $d \hat{s}^{2}$ by $e^{2 a \sigma}$, after which one finds, for vanishing asymptotic values of $\sigma$ and $\rho$, the solution

$$
\begin{aligned}
d s^{2} & =e^{2 a \sigma} d \hat{s}^{2} \\
& =-\left(1-\frac{\left(1+a^{2}\right) M}{r}\right)^{\frac{2\left(1-a^{2}\right)}{\left(1+a^{2}\right)}} d t^{2}+\left(1-\frac{\left(1+a^{2}\right) M}{r}\right)^{-2} d r^{2}+r^{2} d \Omega_{2}^{2} \\
e^{-a \sigma} & =\left(1-\frac{\left(1+a^{2}\right) M}{r}\right)^{\frac{a^{2}}{1+a^{2}}}, \quad \rho=0
\end{aligned}
$$

where $M$ is the ADM mass and $d \Omega_{2}^{2}$ is the metric on the unit 2-sphere. When $a=1$ and $\sigma$ is the dilaton field this rescaling of the canonical metric is exactly what is required to get the so-called 'string metric', so that the $a=1$ black holes have a natural interpretation as string solitons. This might make it appear that the rescaling of the canonical metric by $e^{2 a \sigma}$ is inappropriate to string theory when $a \neq 1$, but it must be remembered that the scalar field $\sigma$ is not necessarily the dilaton but is, in general, a combination of the dilaton and modulus fields of the torus and gauge fields. Indeed, it was shown in [37] that for the $a=\sqrt{3}$ black holes this combination is such that the effective rescaling is just that of (3.2). For 
any value of $a$ this metric has an internal infinity as $r \rightarrow\left(1+a^{2}\right) M$ for constant $t$. For $a<1$ the surface $r=\left(1+a^{2}\right) M$ is an event horizon, but this event horizon is regular only if $2\left(1-a^{2}\right) /\left(1+a^{2}\right)$ is an integer, which restricts the values of $a$ less than unity to $a=0$ or $a=1 / \sqrt{3}$. The $a=0$ case is the extreme RN black hole for which the soliton interpretation is widely accepted. The significance of the $a=1 / \sqrt{3}$ case has been explained in [38]. For $a \geq 1$ the surface $r=\left(1+a^{2}\right) M$ is at infinite affine parameter along any geodesic so one might admit all values of $a \geq 1$. On the other hand, the relevance of geodesic completeness is not clear in this context so one might still wish to insist that $2\left(1-a^{2}\right) /\left(1+a^{2}\right)$ be an integer so that the null surface $r=\left(1+a^{2}\right) M$ is regular, in which case only the further values of $a=1$ and $a=\sqrt{3}$ can be admitted. Curiously, the values

$$
a=0, \frac{1}{\sqrt{3}}, 1, \sqrt{3}
$$

which we find in this way by demanding that the solution (3.2) is a bone fide soliton also arise from truncation of $N=8$ supergravity. The possibility of the values $a=0$ and $a=1$ is guaranteed by the existence of consistent truncations of $N=8$ supergravity to $N=2$ and $N=4$ supergravity respectively. The possibility of the values $a=\sqrt{3}$ and $a=\frac{1}{\sqrt{3}}$ is guaranteed by the existence of a consistent truncation of the maximal five-dimensional supergravity to simple five-dimensional supergravity since the subsequent reduction to four dimensions yields just these values.

Consider first the $a=0$, electric and magnetic extreme RN black holes. Given any one such black hole with integral charge, an infinite number can be generated by acting with $G(\mathbb{Z})$, and these will include black holes carrying each of the 56 types of charge [35], and this is already sufficient to show that the continuous duality group $E_{7(7)}$ is broken to a discrete subgroup. These solutions break $3 / 4$ of the supersymmetry in the $N=4$ theories and $7 / 8$ of the supersymmetry in the $N=8$ case. For the remainder of the paper, we shall restrict ourselves to solitons which break half the supersymmetry, and the only extreme black hole 
solutions of this type are those with $a=\sqrt{3}$. This follows from consideration of the implications of supersymmetry for the moduli space of multi-black hole solutions. This multi-soliton moduli space is the target space for an effective sigma model describing non-relativistic solitons [39]. This sigma model must have 8 supersymmetries for solitons of a four-dimensional $N=4$ supergravity theory that break half the supersymmetry, and this implies that the moduli space is hyperKahler. Similarly, the moduli space for multi-solitons of $N=8$ supergravity that break half the supersymmetry is the target space for a sigma model with 16 supersymmetries, and this implies that the moduli space is flat. However, the moduli space of multi-black hole solutions is flat if and only if $a=\sqrt{3}[40,41]$, so only these extreme black holes can be solutions of $N=8$ supergravity that break half the supersymmetry. An alternative characterization of these extreme black holes is as 'compactifications' of the extreme black p-brane solitons of the tendimensional supergravity theory, which are known to break half the supersymmetry [42]. It follows that the moduli space of these solutions must be flat and what evidence there is [43] confirms this prediction. This ten-dimensional interpretation of the solitons discussed here will be left to the following section where it will also become clear that they carry combinations of all $28+28$ electric and magnetic charges associated with the $28 U(1)$ gauge fields.

This moduli space argument shows, incidentally, that whereas the flatness of the moduli space for solitons that break half the supersymmetry is protected by supersymmetry for $N=8$ supergravity, this is not so for $N=4$ theories. There is then no reason to expect the moduli space metric of extreme black hole solitons of the exact heterotic string theory (to all orders in $\alpha^{\prime}$ and $g$ ) to be flat. Indeed, the $a=\sqrt{3}$ extreme black holes, which have a flat moduli space, are only approximate solutions of the heterotic string and are expected to receive higher order corrections. Furthermore, if BPS-type monopoles were to occur in the type II string theory, a possibility that is suggested by the occurrence of non-abelian gauge groups in some versions of the compactified type II string [44], they would have to break more than half the supersymmetry as their moduli space is not flat. This is in accord with 
the fact that the four-dimensional type II strings of ref. [44] have at most $N=4$ supersymmetry, so that solitons of these theories saturating a Bogomolnyi bound would have less than $N=4$ supersymmetry. This provides further justification for our assumption that the solitons of the toroidally compactified type II string that break half the $N=8$ supersymmetry are those of the effective $N=8$ supergravity theory.

The complete set of soliton solutions of a supergravity theory fill out multiplets of the duality group $G(\mathbb{Z})$, as mentioned in the introduction. We shall now explain this in more detail. Flat four-dimensional space-time with the scalar fields $\phi^{i}$ taking constant values, $\phi_{0}^{i}$, is a vacuum solution of the supergravity theory parameterised by these constants. The duality group acts non-trivially on such vacua as it changes the $\phi_{0}^{i}$. The solitons for which the scalar fields tend asymptotically to the values $\phi_{0}^{i}$ provide the solitonic Bogomolnyi states about the vacuum state $\mid \phi_{0}>$. A $G(\mathbb{Z})$ transformation takes a Bogomolnyi state in this vacuum with charge vector $\mathcal{Z}$ to another Bogomolnyi state with charge vector $\mathcal{Z}^{\prime}$ and equal mass but in a new vacuum $\mid \phi_{0}^{\prime}>$. As in [4], it will be assumed that one can smoothly continue from $\phi_{0}^{\prime}$ to $\phi_{0}$ without encountering a phase transition, to obtain a state with the charge vector $\mathcal{Z}^{\prime}$, but a different mass in general, about the original vacuum $\mid \phi_{0}>$. This assumption seems reasonable because the extreme black hole solutions depend analytically on the constants $\phi_{0}^{i}$. We thus obtain a new Bogomolnyi soliton solution about the original vacuum but with a $G(\mathbb{Z})$ transformed charge vector. The spectrum of all the Bogomolnyi states obtained in this way is $G(\mathbb{Z})$ invariant by construction. In particular, the number of these Bogomolnyi states with charge vector $\mathcal{Z}$ will be the same as the number with charge vector $\mathcal{Z}^{\prime}$ whenever $\mathcal{Z}$ is related to $\mathcal{Z}^{\prime}$ by a $G(\mathbb{Z})$-transformation.

In addition to the Bogomolnyi states that arise from solitons, there are also the electrically charged Bogomolnyi states of the fundamental string. These states are purely perturbative and for the type II string they consist of the Kaluza-Klein $(\mathrm{KK})$ and winding modes of the string. If they are also to fit into multiplets of the duality group they must have magnetically charged partners under duality, and 
these should be non-perturbative, i.e. solitonic. The soliton duality multiplets are, however, already complete for the reason just given. In order to have duality of the string theory we must therefore identify the fundamental states with electrically charged solitonic states. We shall see in the next section how this identification must be made.

It might be thought that all electrically charged soliton states should have an equivalent description in terms of fundamental states. This is presumably true of the heterotic string since there are fundamental string states carrying each of the 28 types of electric charge and these are related by the T-duality group $O(6,22 ; \mathbb{Z})$. In contrast, the fundamental modes of the type II string carry only 12 of the possible 28 electric charges, because the 16 Ramond-Ramond (RR) $U(1)$ gauge fields couple to the string through their field strengths only. The 12 string-mode electric charges are related by the T-duality group $O(6,6 ; \mathbb{Z})$ of the type II string. It would be consistent with $\mathrm{S}$ and T-duality to suppose that there are no charged states coupling to the 16 (RR) gauge fields, but this would not be consistent with U-duality, as we now show.

Recall that an $n$-dimensional representation of $G$ gives an action of $G$ on $\mathbb{R}^{n}$ which restricts to an action of $G(\mathbb{Z})$ on the lattice $\mathbb{Z}^{n}$. For both the heterotic and type II strings, the charge vector $\mathcal{Z}$ transforms under $G$ as a 56-dimensional representation. For the heterotic string, $G=S L(2 ; \mathbb{R}) \times O(6,22)$ and $\mathcal{Z}$ transforms according to its irreducible $(\mathbf{2}, \mathbf{2 8})$ representation. This has the decomposition

$$
(\mathbf{2}, \mathbf{2 8}) \rightarrow(\mathbf{2}, \mathbf{1 2})+16 \times(\mathbf{2}, \mathbf{1})
$$

in terms of representations of $S L(2 ; \mathbb{R}) \times O(6,6)$. This is to be compared with the type II string for which $G=E_{7(7)}$ and $\mathcal{Z}$ transforms according to its irreducible $\mathbf{5 6}$ representation, which has the decomposition

$$
56 \rightarrow(2,12)+(1,32)
$$

under $S L(2 ; \mathbb{R}) \times O(6,6)$. In both cases there is a common sector corresponding to the $(\mathbf{2}, \mathbf{1 2})$ representation of $S L(2 ; \mathbb{R}) \times O(6,6)$, plus an additional 32-dimensional 
representation corresponding, for the heterotic string, to the charges for the additional $U(1)^{16}$ gauge group and, for the type II strings, to the charges for the Ramond-Ramond (RR) sector gauge fields. It is remarkable that the latter fit into the irreducible spinor representation of $O(6,6)$. These decompositions of the 56 representation of $G$ induce corresponding decompositions of representations of $G(\mathbb{Z})$ into representations of $S L(2 ; \mathbb{Z}) \times O(6,6 ; \mathbb{Z})$ on the charge lattice $\mathbb{Z}^{56}$. In particular, $U$-duality requires the $16+16$ electric and magnetic charges of the $R R$ sector to exist and to transform irreducibly under the action of the T-duality group $O(6,6 ; \mathbb{Z})$, and we conclude that all charges in the RR sector must be carried by solitons. We shall later confirm this.

\section{4. $p$-Brane interpretation of Bogomolnyi solitons}

We have seen that the solitons of toroidally compactified superstrings fit into representations of the duality group $G(\mathbb{Z})$. Our concern here will be to identify states that break half the supersymmetry and carry just one of the 56 types of electric or magnetic charge. We shall call such states for which the charge takes the minimum value 'elementary'; acting on these with the duality group $G(\mathbb{Z})$ will generate a lattice of charged states. Here we wish to show how the elementary solitons arise from extreme black $p$-brane solitons of the ten dimensional effective supergravity theory. These may be of electric or magnetic type. Electric $p$-brane solitons give electrically charged solitons of the four-dimensional dimensionally reduced field theory, while magnetic ones give magnetic monopoles, provided we use the form of the four-dimensional supergravity theory that comes directly from dimensional reduction without performing any duality transformations on the oneform gauge fields (although we convert two-form gauge fields to scalar fields in the usual way). If we had chosen a different dual form of action, the solutions would be the same, but some of the electric charges would be viewed as magnetic ones, and vice versa. This form of the action is manifestly invariant under T-duality: for the heterotic string, the action is the $O(6,22)$-invariant one given in [45], which is 
related to the one of [46] by a duality transformation, and for the type II string, it is a new $O(6,6)$-invariant form of the $N=8$ supergravity action which is related to the $S L(8, \mathbb{R})$-invariant Cremmer-Julia action [7] by a duality transformation.

An extreme $p$-brane soliton of the ten-dimensional low-energy field theory has a metric of the form [21]

$$
d s^{2}=A(r)\left[-d t^{2}+d x^{i} d x^{i}\right]+B(r) d r^{2}+r^{2} d \Omega_{8-p}^{2}
$$

where $x^{i}(i=1, \ldots, p)$ are $p$ flat Euclidean dimensions, $d \Omega_{8-p}^{2}$ is the metric on an $(8-p)$-sphere, $r$ is a radial coordinate, $t$ is a time coordinate and $A(r), B(r)$ are two radial functions that tend to unity as $r \rightarrow \infty$. These solitons couple either to an anti-symmetric tensor gauge field $A_{r}$ of rank $r=7-p$, in which case the $p$-brane is magnetically charged and $F=d A$ is proportional to the $(8-p)$-sphere volume form $\epsilon_{8-p}$, or one of rank $r=p-3$, in which case the brane is electric and $\star F$ is proportional to $\epsilon_{8-p}$. In some cases, the $p$-brane solutions will have corrections of higher order in $\alpha^{\prime}$, but some of the solutions correspond to exact conformal field theories.

We shall be interested in four dimensional solitons obtained by 'compactifying' $p$-brane solitons on the six-torus. Compactification on $T^{p}$ is straightforward since one has only to 'wrap' the $p$-brane around the $p$-torus, which is achieved by making the appropriate identifications of the $x^{i}$ coordinates. If $p<6$ a soliton in four dimensions can then be found by taking periodic arrays on $T^{(6-p)}$ and making a periodic identification * For example [47], a five-brane can be wrapped around a five-torus in six ways giving rise to six types of five-dimensional soliton and these yield six types of black-hole solitons in four dimensions on taking periodic arrays. Similarly, to 'compactify' a 0-brane (i.e. a 10-dimensional black hole) on a sixtorus one first introduces a 6-dimensional periodic array of such black holes and

* Alternatively, since the solution of extreme black $p$-branes always reduces to the solution of the Laplace equation in the transverse space, one has only to solve this equation on $\mathbb{R}^{3} \times T^{(6-p)}$ instead of $\mathbb{R}^{(9-p)}$ to find solitons of the four-dimensional theory. 
periodically identifies. Instead of wrapping all $p$ dimensions of a $p$-brane to obtain a point-like 0 -brane in $3+1$ dimensions, one can wrap $p-q$ dimensions to obtain a q-brane soliton in 3+1 dimensions; however, in what follows we shall restrict ourselves to 0 -brane solitons in 4 dimensions.

The bosonic sectors of the ten-dimensional effective field theories of the heterotic and type IIA and type IIB superstrings each include a metric, $g_{M N}$, antisymmetric tensor gauge field, $b_{M N}$, and a dilaton field $\Phi$. We shall first discuss this common sector of all three theories and then turn to the additional sectors characteristic of each theory. We expect the solutions we describe to be exact solutions of the classical type II theory, and their masses to be unrenormalized in the quantum theory, but for the heterotic string they are only approximate solutions (to lowest order in $\alpha^{\prime}$ ) of the low energy field theory.

Dimensional reduction of the common $(g, b, \Phi)$ sector on $T^{6}$ yields 6 KaluzaKlein abelian gauge fields $\left(g_{\mu i}+\cdots\right)$ coming from $g_{M N}$ and another 6 abelian gauge fields $\left(b_{\mu i}+\cdots\right)$ coming from $b_{M N}$. It is straightforward to identify the magnetically-charged solitons associated with the KK gauge fields. These are the KK monopoles [48], consisting of the product of a self-dual Taub-NUT instanton, with topology $\mathbb{R}^{4}$, with a 5-torus and a time-like $\mathbb{R}$. As this is the the product of a five-metric with a five-torus, this can also be viewed as a fivebrane solution of the ten-dimensional theory wrapped around a five-torus. ${ }^{\dagger}$ There are six types of KK monopoles in four dimensions, one for each of the six KK gauge fields, because the fivebrane can be wrapped around the six-torus in six different ways. As fourdimensional solutions the KK monopoles are extreme black holes with $a=\sqrt{3}$, as expected from the moduli space argument of the previous section. The elementary magnetically charged solitons associated with the $b_{\mu i}$ gauge fields can be identified with the six possible 'compactifications' of the extreme black fivebrane $[20,19]$ of the ten dimensional $(g, b, \Phi)$ theory. We shall refer to these as abelian H-monopoles;

$\dagger$ For fixed $r, t$, the solution has topology $S^{3} \times T^{5}$, and the $S^{3}$ can be regarded as a Hopf bundle of $S^{1}$ over $S^{2}$. Thus locally it is $S^{2} \times T^{6}$, so that this solution might also be thought of as a twisted 6-brane. 
they were first given in [49] and have been discussed further in [50,47,22]. It is straightforward to check directly that the KK monopoles and the H-monopoles are indeed related by T-duality, as expected [51,52]. Note that we have not included KK modes of the 5-brane, i.e. configurations in which the 5-brane has momentum in some of the toroidal directions, as these either lead to extended objects in four dimensions, or to localised solitons that carry more than one type of charge and so are not elementary.

The KK and abelian $\mathrm{H}$ monopoles have electric duals. These electrically charged solitons have their ten-dimensional origin in the extreme black string [18] of the $(g, b, \Phi)$ theory, which is dual $[53,54,55]$ to the extreme black five-brane. The 6 electric duals to the abelian H-monopoles are found by wrapping the solitonic string around the 6 -torus, i.e. the 6 winding modes of the solitonic string. The electric duals of the KK monopoles come from Kaluza Klein modes of the 1-brane, i.e. configurations in which the solitonic string has momentum in the toroidal directions. They can be thought of as pp-waves travelling in the compactified directions [11]. These $6+6$ elementary electrically charged solitons couple to the $6+6 \mathrm{KK}$ and $b_{\mu i}$ gauge fields. They are in one to one correspondence with the KK (i.e. torus momentum modes) and winding states of the fundamental string which couple to the same 12 gauge fields. This allows us, in principle, to identify the fundamental string states as soliton states and, as explained in earlier sections, U-duality of string theory forces us to do so.

Before turning to solitons of the additional sector of each string theory, we shall first explain here why these field theory solitons are exact solutions of type II string theory. Type II string theory in a $(g, b, \Phi)$ background is described by a non-linear sigma-model with $(1,1)$ world-sheet supersymmetry. The KK monopole background is described by a $(4,4)$ supersymmetric sigma-model plus a free $(1,1)$ supersymmetric field theory; this is conformally invariant [56] and so gives an exact classical solution of string theory. The pp-wave background is also an exact classical solution [57], so that the T-duals of these two solutions must be exact classical solutions too. In contrast, the heterotic string in a $(g, b, \Phi)$ background 
is described by a $(1,0)$ supersymmetric sigma-model, and at least some of the solutions described above only satisfy the field equations to lowest order in $\alpha^{\prime}$. In some cases, as we will describe later, these solutions can be modified to obtain exact classical heterotic string solutions. However, it is not known in general whether such backgrounds can be modified by higher order corrections to give an exact string solution.

We have now accounted for $12+12$ of the required $28+28$ types of charge of all three ten-dimensional superstring theories. We now consider how the additional $16+16$ charges arise in each of these three theories, starting with the type II string. It is known that, after toroidal compactification, the type IIA and type IIB string theories are equivalent [58] but it is instructive to consider both of them. In either case, we showed in the last section that U-duality requires that the missing $16+16$ types of charge transform as the irreducible spinor representation of the Tduality group. Since T-duality is a perturbative symmetry, if there were electrically charged states of this type in the fundamental string spectrum, there would also have to be magnetic ones. However, magnetic charges only occur in the soliton sector, so a prediction of U-duality is that the corresponding 16 electric charges are also to be found in the soliton sector and not, as one might have thought, in the elementary string spectrum. We shall confirm this.

First we consider the type IIA theory. The ten-dimensional bosonic massless fields are the $(g, b, \Phi)$ fields of the common sector plus a one-form gauge potential, $A_{M}$, and a three-form gauge potential, $A_{M N P}$. These extra fields appear in the RR sector but couple to the string through their field strengths only. Upon compactification to four dimensions, $A_{M}$ gives one abelian gauge field $A_{\mu}$ and $A_{M N P}$ gives 15 abelian gauge fields $A_{\mu}^{i j}$. These also couple to the string through their field strengths only and so there are no elementary string excitations that are electrically-charged with respect to these 16 gauge fields, as expected. The solitonic $p$-brane solutions of the ten-dimensional field theory involving $A_{M}$ or $A_{M N P}$ and breaking only half the supersymmetry consist of a 0 -brane, i.e. a (tendimensional) extreme black hole, a 2-brane (i.e. a membrane), a 4-brane and a 
6-brane. The 0-brane and the 2-brane are of electric type. The 0-brane gives rise to an electrically-charged four-dimensional black hole in the toroidally-compactified theory by the procedure of taking periodic arrays of the ten-dimensional solution. The membrane gives a total of $\frac{6 \times 5}{2}=15$ electric black holes in four dimensions after 'wrapping' it around two directions of the six-torus and then taking periodic arrays to construct a four-dimensional solution. Similarly, the magnetic type 4-brane and 6-brane can be wrapped around the six-torus (introducing periodic arrays where necessary) to give 15+1 magnetically-charged black holes in four dimensions. We have therefore found a total of 32 additional electric and magnetic charges. Combined with the previous 24 charges this gives a total of 56 elementary charged states carrying only one type of charge. From the low-energy field theory we know that these charges transform according to the $\mathbf{5 6}$ representation of $E_{7}$, and that acting on these elementary solitons with $E_{7}(\mathbb{Z})$ generates a 56 -dimensional charge lattice. As anticipated, the extra 16+16 electric and magnetic charges are inert under S-duality but are mixed by the T-duality group $O(6,6 ; \mathbb{Z})$. In addition to the $p$-brane winding modes discussed above, there are also $p$-brane momentum modes; however, to give a 0-brane in 4 dimensions, the $p$-brane must wrap around the torus as well as having internal momentum, so that the resulting soliton would carry more than one type of charge and so would not be elementary; nevertheless, these solitons occur in the charge lattices generated by the elementary solitons.

A similar analysis can be made for the type IIB theory. In this case the extra massless bosonic fields in the ten dimensional effective field theory are a scalar, a two-form potential, $A_{M N}$, and a four-form potential $A_{M N P Q}^{(+)}$with self-dual fiveform field strength. As for the type IIA string theory, these gauge fields couple through their field strengths only and so, again, there are no string excitations carrying the new electric charges. In the solitonic sector of the ten-dimensional field theory there is a neutral 5-brane, a self-dual 3-brane and a string, in addition to the string and neutral fivebrane of the $(g, b, \Phi)$ sector. The new neutral fivebrane gives 6 magnetic charges in four dimensions, the self-dual 3-brane gives 10 electric and 10 magnetic charges and the new string gives six electric charges. Note that 
the new solitonic string couples to the $16 U(1)$ gauge fields coming from $A_{M N}$ and $A_{M N P Q}^{(+)}$. These $16+16$ charges couple to $A_{M N}$, while the fundamental string and the solitonic string of the common sector both couple to $b_{M N}$; thus it may be consistent to identify the fundamental and common sector solitonic strings, but the new solitonic string cannot be identified with either. As for the type IIA string all 56 charges generate the irreducible 56-dimensional representation of $E_{7}(\mathbb{Z})$.

Finally, we turn to the heterotic string. We have seen that the common sector solutions of the low-energy effective supergravity theory include $12 \mathrm{KK}$ and abelian $\mathrm{H}$ monopoles, and their 12 electric duals, and under T-duality these must have $16+16$ electric and magnetic black hole partners coupling to the 16 remaining $U(1)$ gauge fields. These have a ten-dimensional interpretation as the 0-branes and 8-branes of $N=1$ ten-dimensional supergravity coupled to 16 abelian vector multiplets [21] (which can be taken to be those of the $U(1)^{16}$ subgroup of $E_{8} \times E_{8}$ or $\left.S O(32) / \mathbb{Z}_{2}\right)$.

In addition to these black hole solutions, there are also BPS monopole solutions of the heterotic string arising from wrapping heterotic or gauge five-branes around the six-torus [16]. The BPS monopoles are not solutions of the effective supergravity theory with abelian gauge group, but it has been argued (e.g. in [47]) that there should be modifications of these monopoles that are solutions of the abelian theory. The moduli spaces for multi-soliton solutions of BPS monopoles are hyper-Kahler [59] while those for extreme $a=\sqrt{3}$ black holes are flat $[40,41]$, so that the black holes and BPS monopoles should not be related by duality. If the modified BPS monopoles also have a non-flat moduli space, then they too cannot be dual to black holes. However, it is also possible that they have a flat moduli space, and even that they are equivalent to black hole solutions. The modified BPS monopoles, if they exist, would have electric partners under $S$-duality which would be electric solitons. The magnetic partners under S-duality of electrically charged Bogomolnyi fundamental string states are expected to be magnetic monopole solitons, which might be either BPS-type solutions, or black holes (or both, if they are equivalent). In either case, the fundamental string states should be identified with 
the electrically charged solitons related to the magnetic monopoles by S-duality.

Whereas the solutions of the type II string we have discussed are exact conformal field theories, the solutions of the heterotic string are only approximate low-energy solutions. However, some of these heterotic solutions have a compact holonomy group, and for these one can set the Yang-Mills connection equal to the spin-connection so that the sigma-model becomes one with $(1,1)$ supersymmetry and the resulting background is an exact solution of string theory. Applying this to the five-brane gives the symmetric five-brane solution [50] and this can also be used to construct a 'symmetric KK monopole'. However, we do not know which of the other solutions of the low-energy effective theory can be corrected to give exact solutions, and the duality symmetry of non-abelian phases of the theory are not understood. Moreover the $\alpha^{\prime}$ corrections to the four-dimensional supergravity action give a theory that is not S-duality invariant, but if string-loop corrections are also included, an S-duality invariant action should arise.

\section{Toroidal compactification to other dimensions}

In this section, we extend the previous discussion to consider the duality symmetries of type II and heterotic strings toroidally compactified to $d$ dimensions. The resulting low-energy field theory is a $d$-dimensional supergravity theory which has a rigid 'duality' group $G$, which is a symmetry of the equations of motion, and in odd dimensions is in fact a symmetry of the action. In each case the massless scalar fields of the theory take values in $G / H$, where $H$ is the maximal compact subgroup of $G$. $G$ has an $O(10-d, 10-d)$ subgroup for the type II string, and an $O(10-d, 26-d)$ subgroup for the heterotic string. In either string theory, it is known that this subgroup is broken down to the discrete T-duality group, $O(10-d, 10-d ; \mathbb{Z})$ or $O(10-d, 26-d ; \mathbb{Z})$. It is natural to conjecture that the whole supergravity duality group $G$ is broken down to a discrete subgroup $G(\mathbb{Z})$ (defined below) in the $d$-dimensional string theory. We have already seen that this occurs for $d=4$ and will argue that for $d>4$ the symmetry $G$ is broken to a dis- 
crete subgroup by a generalisation of the Dirac quantization condition. In tables 1 and 2, we list these groups for toroidally compactified superstring theories (at a generic point in the moduli space so that the gauge group is abelian).

\begin{tabular}{|c|c|c|c|}
\hline $\begin{array}{c}\text { Space-time } \\
\text { Dimension d }\end{array}$ & Supergravity Duality Group $G$ & String T-duality & $\begin{array}{c}\text { Conjectured } \\
\text { Full String Duality }\end{array}$ \\
\hline $10 A$ & $S O(1,1) / \mathbb{Z}_{2}$ & $\mathbb{1}$ & $S L(2, \mathbb{Z})$ \\
\hline $10 B$ & $S L(2, \mathbb{R})$ & $\mathbb{1}$ & $S L(2, \mathbb{Z}) \times \mathbb{Z} 2$ \\
\hline 9 & $S L(2, \mathbb{R}) \times O(1,1)$ & $\mathbb{Z}_{2}$ & $S L(3, \mathbb{Z}) \times S L(2, \mathbb{Z})$ \\
\hline 8 & $S L(3, \mathbb{R}) \times S L(2, \mathbb{R})$ & $O(3,3 ; \mathbb{Z})$ & $O(5,5 ; \mathbb{Z})$ \\
\hline 7 & $O(5,5)$ & $O(4,4 ; \mathbb{Z})$ & $S L(5, \mathbb{Z})$ \\
\hline 6 & $S L(5, \mathbb{R})$ & $O(5,5 ; \mathbb{Z})$ & $E_{6(6)}(\mathbb{Z})$ \\
\hline 5 & $E_{6(6)}$ & $O(6,6 ; \mathbb{Z})$ & $E_{7(7)}(\mathbb{Z})$ \\
\hline 4 & $E_{7(7)}$ & $O(7,7 ; \mathbb{Z})$ & $E_{8(8)}(\mathbb{Z})$ \\
\hline 3 & $E_{8(8)}$ & $O(8,8 ; \mathbb{Z})$ & $E_{9(9)}(\mathbb{Z})$ \\
\hline 2 & $E_{9(9)}$ & $O(9,9 ; \mathbb{Z})$ & $E_{10(10)}(\mathbb{Z})$ \\
\hline 1 & $E_{10(10)}$ & &
\end{tabular}

Table 1 Duality symmetries for type II string compactified to $d$ dimensions.

For the type II string, the supergravity duality groups $G$ are given in $[60$, $61]^{\star}$. The Lie algebra of $E_{9(9)}$ is the $E_{8(8)}$ Kac-Moody algebra, while the algebra corresponding to the $E_{10}$ Dynkin diagram has been discussed in $[61,63]$. The $d=2$ duality symmetry contains the infinite-dimensional Geroch symmetry group of toroidally compactified general relativity. In $d=9$, the conjectured duality group is a product of an $S L(2, \mathbb{Z})$ S-duality and a $\mathbb{Z}_{2}$ T-duality, while for $d<8$ we conjecture a unified U-duality. For $d=8$, the T-duality group $O(2,2 ; \mathbb{Z}) \sim$ $[S L(2, \mathbb{Z}) \times S L(2, \mathbb{Z})] / \mathbb{Z}_{2} \times \mathbb{Z}_{2}$ is a subgroup of the conjectured duality. In $d=10$, the type IIA string has $G=S O(1,1) / \mathbb{Z}_{2}$, while the type IIB has $G=S L(2, \mathbb{R})$, as indicated in the first two lines of the table. We shall abbreviate $E_{n(n)}(\mathbb{Z})$ to $E_{n}(\mathbb{Z})$ when no confusion can arise.

$\star$ They have also been discussed in the context of worldvolume actions of extended objects in supergravity backgrounds [62]. 


\begin{tabular}{|c|c|c|c|}
\hline $\begin{array}{c}\text { Space-time } \\
\text { Dimension d }\end{array}$ & $\begin{array}{c}\text { Supergravity } \\
\text { Duality Group } G\end{array}$ & String T-duality & $\begin{array}{c}\text { Conjectured } \\
\text { Full String Duality }\end{array}$ \\
\hline 10 & $O(16) \times S O(1,1)$ & $O(16 ; \mathbb{Z})$ & $O(16 ; \mathbb{Z}) \times \mathbb{Z}_{2}$ \\
\hline 9 & $O(1,17) \times S O(1,1)$ & $O(1,17 ; \mathbb{Z})$ & $O(1,17 ; \mathbb{Z}) \times \mathbb{Z}_{2}$ \\
\hline 8 & $O(2,18) \times S O(1,1)$ & $O(2,18 ; \mathbb{Z})$ & $O(2,18 ; \mathbb{Z}) \times \mathbb{Z}_{2}$ \\
\hline 7 & $O(3,19) \times S O(1,1)$ & $O(3,19 ; \mathbb{Z})$ & $O(3,19 ; \mathbb{Z}) \times \mathbb{Z}_{2}$ \\
\hline 6 & $O(4,20) \times S O(1,1)$ & $O(4,20 ; \mathbb{Z})$ & $O(4,20 ; \mathbb{Z}) \times \mathbb{Z}_{2}$ \\
\hline 5 & $O(5,21) \times S O(1,1)$ & $O(5,21 ; \mathbb{Z})$ & $O(5,21 ; \mathbb{Z}) \times \mathbb{Z} 2$ \\
\hline 4 & $O(6,22) \times S L(2, \mathbb{R})$ & $O(6,22 ; \mathbb{Z})$ & $O(6,22 ; \mathbb{Z}) \times S L(2, \mathbb{Z})$ \\
\hline 3 & $O(8,24)$ & $O(7,23 ; \mathbb{Z})$ & $O(8,24 ; \mathbb{Z})$ \\
\hline 2 & $O(8,24)^{(1)}$ & $O(8,24 ; \mathbb{Z})$ & $O(8,24)^{(1)}(\mathbb{Z})$ \\
\hline
\end{tabular}

Table 2 Duality symmetries for heterotic string compactified to $d$ dimensions.

For the heterotic string, the supergravity duality groups $G$ for $d>2$ can be found in articles collected in [64]. Pure $N=4$ supergravity in $d=4$ reduces to a theory with $G=S O(8,2)$ in $d=3$ and to a theory with supergravity duality group given by the affine group $S O(8,2)^{(1)}$ in $d=2$ [60]. Similar arguments suggest that the heterotic string should give a $d=2$ supergravity theory with $G$ given by the affine group $O(8,24)^{(1)}$ symmetry. The heterotic string is conjectured to have an $S \times T$ duality symmetry in $d \geq 4$ and a unified U-duality in $d \leq 3$. Sen conjectured an $O(8,24 ; \mathbb{Z})$ symmetry of $d=3$ heterotic strings in [65]. The $d=10$ supergravity theory has an $O(16)$ symmetry acting on the 16 abelian gauge fields which is broken to the finite group $O(16 ; \mathbb{Z})$; we refer to this as the T-duality symmetry of the ten-dimensional theory.

The supergravity symmetry group $G$ in $d$ dimensions doesn't act on the $d$ dimensional space-time and so survives dimensional reduction. Then $G$ is necessarily a subgroup of the symmetry $G^{\prime}$ in $d^{\prime}<d$ dimensions and dimensional reduction gives an embedding of $G$ in $G^{\prime}$, and $G(\mathbb{Z})$ is a subgroup of $G^{\prime}(\mathbb{Z})$. We use this embedding of $G$ into the duality group in $d^{\prime}=4$ dimensions to define the duality group $G(\mathbb{Z})$ in $d>4$ dimensions as $G \cap E_{7}(\mathbb{Z})$ for the type II string and as $G \cap[O(6,22 ; \mathbb{Z}) \times S L(2, \mathbb{Z})]$ for the heterotic string.

The symmetries in $d<4$ dimensions can be understood using a type of argu- 
ment first developed to describe the Geroch symmetry group of general relativity and used in [65] for $d=3$ heterotic strings. The three-dimensional type II string can be regarded as a four-dimensional theory compactified on a circle and so is expected to have an $E_{7}(\mathbb{Z})$ symmetry. There would then be seven different $E_{7}(\mathbb{Z})$ symmetry groups of the three dimensional theory corresponding to each of the seven different ways of first compactifying from ten to four dimensions, and then from four to three. The seven $E_{7}(\mathbb{Z})$ groups and the $O(7,7 ; \mathbb{Z})$ T-duality group do not commute with each other and generate a discrete subgroup of $E_{8}$ which we define to be $E_{8}(\mathbb{Z})$. (Note that the corresponding Lie algebras, consisting of seven $E_{7(7)}$ algebras and an $O(7,7)$, generate the whole of the $E_{8(8)}$ Lie algebra.) Similarly, in $d=2$ dimensions, there are eight $E_{8}(\mathbb{Z})$ symmetry groups and an $O(8,8 ; \mathbb{Z})$ T-duality group which generate $E_{9}(\mathbb{Z})$ as a discrete subgroup of $E_{9(9)}$, and in the heterotic string there are eight $O(8,24 ; \mathbb{Z})$ symmetry groups from three dimensions and an $O(8,24 ; \mathbb{Z})$ T-duality group which generate $O(8,24 ; \mathbb{Z})^{(1)}$ as a discrete subgroup of $O(8,24)^{(1)}$.

We now turn to the charge quantization condition and soliton spectrum in $d>4$ dimensions. Consider first the example of type II string theory compactified to $d=5$ dimensions. The low-energy theory is $d=5, N=8$ supergravity [66] which has 27 abelian vector gauge fields $A_{\mu}^{I}$ and an $E_{6(6)}$ rigid symmetry of the action. Recall that in five dimensions electric charge can be carried by particles or 0-brane solitons, while magnetic charge can be carried by strings or 1-brane solitons. The 27 types of electric charge $q_{I}$ transform as a 27 of $E_{6(6)}$ while 27 types of magnetic charge $p^{I}$ transform as a $\overline{\mathbf{2 7}}$. These charges satisfy the quantization condition $q_{I} p^{I}=$ integer $[53,67]$ which is invariant under $E_{6(6)}$. As we shall see, all 54 types of charge occur and so the electric charges take values in a 27 -dimensional lattice $\Lambda$ and the magnetic ones take values in the dual lattice. This breaks the $E_{6(6)}$ symmetry down to the discrete subgroup which preserves the lattice. If the theory is now compactified to four dimensions, $E_{6(6)}$ survives as a subgroup of the $E_{7}(7)$ duality symmetry in $d=4$ and the 27 -dimensional lattice $\Lambda$ survives as a sub-lattice of the 28-dimensional lattice of $d=4$ electric charges (this will be 
checked for the elementary charged solitons below). Thus the subgroup of $E_{6(6)}$ preserving $\Lambda$ is $E_{6(6)} \cap E_{7}(\mathbb{Z})$, which is precisely the discrete group $E_{6}(\mathbb{Z})$ defined above.

The five-dimensional theory has a Bogomolnyi bound involving the electric and magnetic charges $[11,17]$ which is saturated by Bogomolnyi solutions that do not break all the supersymmetries, and the masses and charges of these states are expected to be unrenormalised in the quantum theory. This bound is invariant under $E_{6(6)}$ and $E_{6}(\mathbb{Z})$ and so Bogomolnyi solitons automatically fit into $E_{6}(\mathbb{Z})$ representations.

The $d=5$ elementary solitons of the type IIA theory carrying precisely one type of electric or magnetic charge and breaking half the supersymmetry can be identified in a similar manner to that used in $d=4$. The 27 elementary electrically charged solitons, which are all extreme black hole solutions in $d=5$, and the 27 magnetic ones, which are all extreme black strings, arise from $d=10$ solutions as follows. The 5 -brane wrapped around the 5 -torus gives one electrically charged 0 -brane and 5 magentically charged strings. The $d=10$ solitonic string gives 5 electric black holes and 1 black string. The 0-brane gives one black hole, the 4-brane gives 10 black holes and 5 black strings, the 4-brane gives 5 black holes and 10 black strings and the 6-brane gives 1 black string. In addition, there are 5 electric black holes arising from pp-waves travelling in each of the 5 toroidal dimensions (these can also be viewed as momentum modes of the solitonic string), and their 5 magnetic duals, which are a magnetic string generalisation of the KK magnetic monopole. These are the solutions consisting of the product of a 4-torus with self-dual Taub-NUT and two-dimensional Minkowski space. The non-compact six-dimensional subspace gives rise to a 5 -dimensional magnetic string in the same way that a 4-dimensional KK magnetic monopole originates from a five-dimensional solution. Note that these solutions can be thought of as wrapped 4-brane solutions. This gives the $27+27$ elementary charges, as required.

On further compactification to $d=4$, the 27 electric charges give 27 electri- 
cally charged black holes in $d=4$ and the magnetic strings give 27 magnetic black holes (together with $27 d=4$ black strings). There are two additional elementary charged states in $d=4$, the pp-wave travelling in the fifth dimension and the KK monopole corresponding to the fifth dimension; these two solutions are uncharged from the five-dimensional point of view. This corresponds to the fact that the four-dimensional charges lie in a $\mathbf{5 6}$ of $E_{7(7)}$ and this decomposes into $E_{6(6)}$ representations as $\mathbf{5 6} \rightarrow \mathbf{2 7}+\overline{\mathbf{2 7}}+\mathbf{1}+\mathbf{1}$.

Similar arguments apply to other strings in $d \geq 4$ dimensions, where charge quantization effects break $G$ to at most the string duality groups listed in the tables. In $d$ dimensions there are electric point charges and magnetic $(d-4)$-brane solitons (which correspond to a subset of the four-dimensional black hole solitons on compactification) and the Dirac quantization of their charges [53,67] breaks the duality symmetry to the discrete subgroup $G(\mathbb{Z})$. There is a similar charge quantization condition on electric $p$-branes and magnetic $d-p-4$ branes in $d$ dimensions $[53,67]$ which again break $G$ to $G(\mathbb{Z})$. In each case, the Bogomolnyi solitons automatically fit into representations of the duality group (providing one can continue solutions from one vacuum to another as discussed in section 3 ).

For $d<4$, it is not clear how to understand the breaking of $G$ to $G(\mathbb{Z})$ directly in terms of $d$-dimensional quantum effects. Thus, while we have shown that for $d \geq 4$ the group $G$ is broken to at most $G(\mathbb{Z})$, there is less evidence for our conjectures for $d<4$, although we do know that a subgroup of $G$ is broken to the discrete T-duality group, and that the solitons will fit into representations of $G(\mathbb{Z})$. 


\section{Compactification of Type II strings on $K_{3} \times T^{2}$}

The analysis of Bogomolnyi states can also be carried out for non-toroidal compactifications. An interesting example is compactification of the type II superstring on $K_{3} \times T^{2}$ because while $K_{3}$ has no non-trivial one-cycles and hence no string winding modes it does have 22 non-trivial two-cycles around which a $p$ brane for $p>1$ can wrap itself to produce a $(p-2)$-brane which will then produce monopole winding states on $T^{2}$ if $p<5$ (taking periodic arrays where necessary). The effective four-dimensional supergravity can be found by the two stage process of compactification to six dimensions on $K_{3}$ [25], followed by a straightforward reduction on $T^{2}$. It is an $N=4$ supergravity with an $S L(2 ; \mathbb{R}) \times O(6,22)$ symmetry and $28 U(1)$ gauge fields, exactly as for the compactification of the heterotic string on $T^{6}$ at a generic point in the moduli space. In fact, the four-dimensional supergravity theories are identical because the coupling of $N=4$ supergravity to $k$ abelian vector multiplets is uniqely determined by the choice of gauge group $[46]^{\star}$. The analysis of section 2 again applies, with the result that the duality group is broken down to $S L(2 ; \mathbb{Z}) \times O(6,22 ; \mathbb{Z})$ by the charge quantization condition, and the Bogomolnyi bound is again duality invariant. The soliton spectrum then automatically fits into representations of $S L(2 ; \mathbb{Z}) \times O(6,22 ; \mathbb{Z})$ and the solitons correspond to precisely to the same extreme black holes as were discussed in section 4 for the heterotic string. However, the ten-dimensional origin of the elementary charged solutions is now different and we now discuss these.

Consider first the common $(g, b, \Phi)$ sector. Because $K_{3}$ has no isometries and no non-trivial one-cycles all KK modes and string winding modes arise from the $T^{2}$ compactification. This yields modes carrying $2+2$ types of electric charge which couple to the $2+2$ gauge fields from the metric and antisymmetric tensor. The corresponding magnetic charges are the KK monopoles and the H-monopoles. The

\footnotetext{
$\star$ Compactification of the heterotic string on $K_{3} \times T^{2}$ leads to a four-dimensional effective field theory with only $N=2$ supersymmetry, for which the masses of the Bogomolnyi states might be expected to receive quantum corrections, so we shall not discuss this case here.
} 
latter can be interpreted as the winding modes on $T^{2}$ of the six-dimensional solitonic string found from 'wrapping' the neutral ten-dimensional fivebrane around the $K_{3}$ surface [68]. We have therefore identified the modes carrying just one type of the $4+4$ electric and magnetic charges in this sector. As before, we do not consider modes arising from the ten-dimensional solitonic string on the grounds that these are not independent of the fundamental string modes already considered.

Consider now the type IIA string. The additional 24 vector gauge fields in the four dimensional effective field theory arise from the ten-dimensional RR gauge fields $A_{M}$ and $A_{M N P}$. One of these vector gauge fields, $A_{\mu}$, is the four-dimensional component of $A_{M}$. The remaining 23 come from expressing the ten-dimensional three-form $A_{M N P}$ as the exterior product of a four-dimensional one-form gauge potential times each of the $22+1$ harmonic two-forms of $K_{3} \times T^{2}$. We must now find the charged Bogomolnyi states to which these fields couple. Again we consider states carrying only one type of charge. The ten-dimensional 0-brane and six-brane solitons associated with $A_{M}$ yield, respectively, one electric and one magnetic fourdimensional black hole coupling to $A_{\mu}$. The electric 2-brane and the magnetic 4brane solitons in ten dimensions produce the Bogomolnyi states carrying the other $23+23$ types of charge coupling to the other 23 gauge fields. Specifically, the 2brane can be wrapped around the $22+1$ non-trivial two-cycles of $K_{3} \times T^{2}$ to produce $22+1$ six-dimensional black holes of which one can then take periodic arrays to get $22+1$ four-dimensional electric black holes. The four brane can be wrapped around the 22 homology two-cycles of $K_{3}$ to give 22 six-dimensional 2-branes, each of which can then be wrapped around $T^{2}$ to produce a four-dimensional magnetic black hole. Alternatively, the four-brane can be wrapped entirely around $K_{3}$ to give one six-dimensional black hole which then produces a further magnetic black hole in four dimensions on taking periodic arrays. We have now found a total of $24+24$ additional electric and magnetic black holes. They each satisfy the Bogomolnyi bound, because the ten-dimensional $p$-brane solitons do, and they each carry just one type charge. Combining these with the $4+4$ black holes from the $(g, b, \Phi)$ sector yields a total of $28+28$ elementary electric and magnetic extreme black holes which 
generate the $(\mathbf{2}, \mathbf{2 8})$ representation of $S L(2 ; \mathbb{Z}) \times O(6,22 ; \mathbb{Z})$.

Consider instead the type IIB superstring. The RR gauge fields are $A_{M N}$ and $A_{M N P Q}^{(+)}$which produce $2+22$ four dimensional gauge fields upon compactification on $K_{3} \times T^{2}$ [25]. These fields couple to the soliton states in four dimensions obtained by wrapping the extra solitonic string and fivebrane, and the self-dual three-brane, around the homology cycles of $K_{3} \times T^{2}$, taking periodic arrays when necessary to get a four-dimensional soliton (alias extreme black hole). There are two homology one-cycles and two homology five-cycles so the extra solitonic string and fivebrane produce $2+2$ four-dimensional electric and magnetic black holes. There are 44 three-cycles so the threebrane produces 44 four-dimensional solitons. Since the threebrane is self-dual 22 of these are electric and 22 magnetic. Again we have a total of $24+24$ additional charges. Combining these with the $4+4$ black holes from the $(g, b, \Phi)$ sector again yields a total of $28+28$ elementary electric and magnetic extreme black holes which generate the $(\mathbf{2 , 2 8})$ representation of $S L(2 ; \mathbb{Z}) \times O(6,22 ; \mathbb{Z})$.

Since the type II string compactified on $K_{3} \times T^{2}$ and the generic toroidal compactification of the heterotic string have exactly the same four-dimensional low-energy field theory it is natural to conjecture that they might be equivalent string theories. If this is so then the Bogomolnyi states of the heterotic string discussed at the conclusion of the previous section would have a straightforward ten-dimensional interpretation after all. It would have some other remarkable consequences. For example, at special points of the heterotic string moduli space, there are extra massless fields and an enhanced (Yang-Mills) symmetry due to nonperturbative world-sheet effects (i.e. non-perturbative in $\alpha^{\prime}$, but perturbative in $g$ ). If the compactified type II string is equivalent, it must have the same enhanced symmetry in vacua corresponding to the same points in the scalar field coset space. This presumably does not arise from non-perturbative world-sheet effects, so would have to come from non-perturbative stringy effects, or from Wilson lines and their $p$-brane generalisations. This would mean that the sigma-model coupling constant $\alpha^{\prime}$ of the heterotic string becomes one of the stringy coupling constants of the 
type II theory, as might have been expected from the fact that for the toridally compactified type II string, all coupling constants are on an equal footing and are mixed up under U-duality.

\section{U-duality and the 11-dimensional supermembrane}

As we have seen, the $E_{7}(\mathbb{Z})$ invariance of the spectrum of soliton states of $N=8$ supergravity is an automatic consequence of the $E_{7}(\mathbb{Z})$ invariance of the equations of motion. The non-trivial features are, firstly, that these states have an interpretation in terms of ten-dimensional KK solitons and solitonic $p$-branes and, secondly, that if the ten-dimensional field theory is considered to be the effective field theory of the type II string theory then $E_{7}(\mathbb{Z})$ invariance requires an identification of the solitonic string with the fundamental string. $N=8$ supergravity can also be obtained by dimensional reduction of 11-dimensional supergravity on $T^{7}$. We shall now show that the elementary soliton states of $N=8$ supergravity also have an interpretation in terms of KK solitons and solitonic $p$-branes of 11-dimensional supergravity. There are $7 \mathrm{KK}$ magnetic monopoles and 7 electric duals, which are pp-waves of 11-dimensional supergravity [69] travelling in the internal dimensions. The 11-dimensional solitonic $p$-branes are the electric membrane and the magnetic fivebrane. Each can be wrapped around the seven-torus to produce 21 four-dimensional solitons. Thus we have a total of 28 electric and 28 magnetic four-dimensional solitons each carrying one of the 56 types of charge which are a basis for the irreducible $\mathbf{5 6}$ repesentation of $E_{7}(\mathbb{Z})$. If we now wish to interpret 11-dimensional supergravity as an effective field theory of a fundamental $E_{7}(\mathbb{Z})$ supermembrane theory then we must identify the fundamental membrane with the solitonic one, just as we were forced to identify the fundamental string with the (appropriate) solitonic string.

Consider now the compactification to $\mathcal{M}_{4}$ on $K_{3} \times T^{3}$ of eleven-dimensional supergravity [28]. The effective field theory is the same as that of the type II superstring compactified on $K_{3} \times T^{2}$, i.e. an $N=4$ supergravity with $28 U(1)$ 
gauge fields and an $S L(2 ; \mathbb{Z}) \times O(6,22 ; \mathbb{Z})$ symmetry. The soliton spectrum is also the same. In the monopole sector we have, firstly, $3 \mathrm{KK}$ monopoles from the $T^{3}$ factor and, secondly, a further 25 monopoles from wrapping the solitonic fivebrane around the $3+22$ homology five-cycles of $K_{3} \times T^{2}$. This gives a total of 28 monopoles. The 28 electrically charged solitons are the electric duals of these monopoles which can be understood in terms of the KK and winding modes of either the solitonic membrane or a fundamental membrane. The entire set of 56 states can be assigned to the $(2,28)$ representation of $S L(2 ; \mathbb{R}) \times O(6,22)$, inducing a corresponding representation of $S L(2 ; \mathbb{Z}) \times O(6,22 ; \mathbb{Z})$.

These results are encouraging signs that it may be possible to define the quantum supermembrane theory entirely in terms of the solitonic membrane solution of eleven-dimensional supergravity. Alternatively, one can envisage a dual formulation in terms of a fundamental 11-dimensional the superfivebrane, in which case the solitonic fivebrane might be identified with a fundamental fivebrane. Of possible relevance in this connection is the fact that the membrane and fivebrane solitons have a very different global structure. Both have a degenerate Killing horizon but whereas the membrane horizon conceals a singularity in an interior region [70], much like the extreme Reissner Nordstrom solution of four-dimensional Maxwell-Einstein theory, the fivebrane is completely non-singular [38].

\section{Comments}

The equations of motion of four-dimensional effective supergravity theories of compactified superstring theories are invariant under a continuous duality group $G$ that is broken by quantum effects to a discrete subgroup $G(\mathbb{Z})$. For the toroidallycompactified heterotic string at a generic point in the moduli space, and for the $K_{3} \times T^{2}$-compactified type II superstrings, this group is the $S \times T$ duality group $S L(2 ; \mathbb{Z}) \times O(6,22 ; \mathbb{Z})$. For the toroidally-compactified type II superstrings it is the $U$ duality group $E_{7}(\mathbb{Z})$ which contains the $S \times T$ duality group $S L(2 ; \mathbb{Z}) \times$ $O(6,22 ; Z)$. Whereas T-duality is known to be an exact symmetry of string theory 
at each order in the string coupling constant $g$, the conjectured S- and T-dualities are non-perturbative. We have provided evidence for U-duality of the type II string by considering those features of string theory that are expected to be given exactly by a semi-classical analysis although it should be emphasized that this evidence depends only on the form of the effective supergravity theory and would apply equally to any consistent quantum theory of gravity for which this is the effective low-energy action. Nevertheless, by supposing this consistent quantum theory to be string theory our arguments have led us to the remarkable conclusions that it is necessary to identify certain states of the string with extreme black holes, and the fundamental string with a solitonic string. We have also seen that the elementary Bogomolnyi states are extreme black hole solutions of the low-energy theory, and have shown how these arise from $p$-brane solitons of the ten-dimensional theory.

The zero modes of the scalar fields of the low energy field theory are all coupling constants of the string theory, so that $G(\mathbb{Z})$ symmetry relates different regimes in the perturbation theory in these coupling constants, interchanging strong and weak coupling and, in the case of $U$-duality, interchanging $g$ with $\alpha^{\prime}$, in the sense of mixing the quantum loop expansion in $g$ with the sigma-model perturbation expansion in $\alpha^{\prime}$ and the moduli of the compactification space. For the compactified type II superstring, any physical quantity (e.g. the S-matrix) can be expanded in terms of the 70 coupling constants associated with zero modes of the 70 scalars. In the world-sheet approach to string theory, one first integrates over the sigmamodel degrees of freedom on a Riemann surface of fixed genus, obtaining a result parameterised by the sigma-model coupling constants, and then sums over genus. As U-duality mixes up all 70 coupling constants, the final result may be expected to depend on all 70 scalars in a symmetric way, even though the calculation was very asymmetrical and in particular picked out the dilaton to play a special role. This would hugely constrain the theory and the assumption of U-duality, together with $N=8$ supersymmety, gives us a great deal of non-perturbative information, and might even enable us to solve the theory! This structure also suggests that there might be a new formulation of string theory which treats all the coupling 
constants on an equal footing.

We have seen that there are duality symmetries in all dimensions $d \leq 10$ and it is interesting to ask whether the $d<10$ symmetries can correspond to symmetries of the 10-dimensional theory. If e.g. the four-dimensional dualities correspond to symmetries of the full ten-dimensional theory, these symmetries must interchange the various $p$-brane solitons of the string-theory; such symmetries would probably have to be non-local. If the theories in $d<4$ dimensions really do have the duality symmetries suggested in section 5, and if these have analogues in higher dimensions, this would have remarkable consequences for string theory. For example, the U-duality of the three-dimensional heterotic string includes transformations that would mix the string with 5-brane solitons in ten dimensions, and so would contain the transformations described as the 'duality of dualities' in [3]. For the type II string theory, this suggests that $E_{10}(\mathbb{Z})$ might be a discrete non-local symmetry of the ten-dimensional string theory!

One of the predictions of S-duality for the heterotic string is the presence of certain dyon bound states in the Bogomolnyi spectrum, which can be translated into to a prediction concerning harmonic forms on the multi-monopole moduli space [71]. It would be interesting to consider the corresponding predictions for the type II string. Recently, some strong-coupling evidence for S-duality of the heterotic string has been found by studying partition functions of certain topological field theories [72], and again it would be interesting to seek similar strong-coupling tests for U-duality.

Finally, since $N=8$ supergravity and its soliton spectrum are U-duality invariant, many of the properties previously thought to be unique to string theory are in fact already properties of the effective supergravity theory once account is taken of all soliton solutions. Since only stable states can appear in an exact S-matrix it is possible that the only states of the exact toroidally compactified type II string theory are the Bogomolnyi states and that these are in one to one correspondence with soliton states of the supergravity theory. This would support 
a previous suggestion [73] that a fundamental superstring theory might actually be equivalent to its effective field theory once solitons of the latter are taken into account. To pursue this further one would need to find higher spin $(>2)$ soliton states corresponding to the higher spin states of string theory. There seems to be no problem in principle with the existence of such higher-spin soliton states in $N=8$ supergravity because the $a=0$ and $a=1$ extreme black holes must belong to massive supermultiplets with spins $>2$ as they break more than half the supersymmetry. In this connection it is worth recalling the similarity of the mass/spin relation for Regge trajectories in four-dimensional string theory, $M^{2} \propto \frac{J}{\alpha^{\prime}}$, with that of the degenerate Kerr solutions of general relativity, $M^{2} \propto \frac{J}{G}$.

Acknowledgements: We are grateful to Louise Dolan, Michael Duff, José Figueroa-O'Farrill, Jerome Gauntlett, Gary Gibbons, Michael Green, Bernard Julia, Wafic Sabra, Trevor Samols, Kellogg Stelle and Edward Witten for helpful comments.

\section{REFERENCES}

1. A. Giveon, M. Porrati and E. Rabinovici, Phys. Rep. 244 (1994) 77.

2. A. Font, L. Ibanez, D. Lust and F. Quevedo, Phys. Lett. B249 (1990) 35;

S.J. Rey, Phys. Rev. D43 (1991) 526.

3. J.H. Schwarz and A. Sen, Nucl. Phys. B411 (1994) 35; Phys. Lett. 312B (1993) 105.

4. A. Sen, Nucl. Phys. B404 (1993) 109; Phys. Lett. 303B (1993); Int. J. Mod. Phys. A8 (1993) 5079; Mod. Phys. Lett. A8 (1993) 2023.

5. K.S. Narain, Phys. Lett. B169 (1986) 41.

6. K.S. Narain, M.H. Sarmadi and E.Witten, Nucl. Phys. B279 (1987) 369.

7. E. Cremmer and B. Julia, Phys. Lett. 80B (1978) 48; Nucl. Phys. B159 (1979) 141. 
8. G.W. Gibbons and C.M. Hull, Phys. Lett. 109B (1982) 190.

9. G.W. Gibbons, in Supersymmetry, Supergravity and Related Topics, eds. F. del Aguila, J. A. de Azcárraga and L.E. Ibañez, (World Scientific 1985).

10. R. Kallosh and T. Ortin, Phys.Rev. D48 (1993) 742.

11. G.W. Gibbons and M.J. Perry, Nucl. Phys. B248 (1984) 629.

12. S. W. Hawking, Monthly Notices Roy. Astron. Soc. 152 (1971) 75; Abdus Salam in Quantum Gravity: an Oxford Symposium (Eds. Isham, Penrose and Sciama, O.U.P. 1975); G. t’Hooft, Nucl. Phys. B335 (1990) 138.

13. M. J. Duff, R. R. Khuri, R. Minasian and J. Rahmfeld, Nucl. Phys. B418 (1994) 195.

14. L. Susskind, preprint hep-th/9309145; J.G. Russo and L. Susskind, preprint hep-th/9405117.

15. M.J. Duff and J. Rahmfeld, preprint hep-th/9406105.

16. J. Harvey, J. Liu, Phys. Lett. B268 (1991) 40.

17. G.W. Gibbons, D. Kastor, L. London, P.K. Townsend and J. Traschen, Nucl. Phys. B416 (1994) 850.

18. A. Dabholkar, G.W. Gibbons, J.A. Harvey and F. Ruiz-Ruiz, Nucl. Phys. B340 (1990) 33; M.J. Duff, G.W. Gibbons and P.K. Townsend, Phys. Lett. 332B (1994) 321.

19. C. Callan, J. Harvey and A. Strominger, Nucl. Phys. B359 (1991) 611.

20. M.J. Duff and J.X. Lu, Nucl. Phys. B354 (1991) 141; Phys. Lett. 273B (1991) 409.

21. G.T. Horowitz and A. Strominger, Nucl. Phys. B360 (1991) 197.

22. M.J. Duff and J.X. Lu, Nucl. Phys. B416 (1993) 301.

23. J. A. de Azcárraga, J.P. Gauntlett, J.M. Izquierdo and P.K. Townsend, Phys. Rev. Lett. 63 (1989) 2443. 
24. E. Witten, Phys. Lett. 153B (1985) 243.

25. P.K. Townsend, Phys. Lett. 139B (1984) 283; M.B. Green, J.H. Schwarz and P.C. West, Nucl. Phys. B254 (1985) 327.

26. E. Bergshoeff, E. Sezgin and P.K. Townsend, Phys. Lett. 189B (1987) 75; Ann. Phys. (N.Y.) 185 (1988) 330.

27. E. Cremmer, B. Julia and J. Scherk, Phys. Lett. 76B (1978) 409.

28. M.J. Duff, B.E.W. Nilsson and C.N. Pope, Phys. Lett 129B (1983) 39.

29. M.J. Duff and K.S. Stelle, Phys. Lett. 253B (1991) 113.

30. R. Güven, Phys. Lett. 276B (1992) 49.

31. M.K. Gaillard and B. Zumino, Nucl. Phys. B193 (1981) 221.

32. M. Günaydin, G. Sierra and P.K. Townsend, Phys. Lett. 133B (1983) 72; Nucl. Phys. B242 (1984) 244.

33. E. Witten, Phys. Lett. 86B (1979) 283.

34. E. Witten and D. Olive, Phys. Lett. B78 (1978) 97.

35. C.M. Hull, Ph.D. Thesis, Cambridge 1983.

36. G.W. Gibbons, Nucl. Phys. B207 (1982) 337; G.W. Gibbons and K. Maeda, Nucl. Phys. B298 (1988) 741; D. Garfinkle, G.T. Horowitz and A. Strominger, Phys. Rev. D43, (1991) 3140; C.F. Holzhey and F. Wilczek, Nucl. Phys. B380 (1992) 447; R. Kallosh, A. Linde, T. Ortin, A. Peet, A. Van Proeyen, Phys.Rev. D46 (992) 5278.

37. M.J. Duff, R.R. Khuri, R. Minasian and J. Rahmfeld, Nucl. Phys. B418 (1994) 195.

38. G.W. Gibbons, G.T. Horowitz and P.K. Townsend, Higher-dimensional resolution of dilatonic black hole singularities, Class. Quantum Grav. in press.

39. J. Gauntlett, Nucl. Phys. B400 (1993) 103. 
40. P. Ruback, Commun. Math. Phys. 107 (1986) 93.

41. K. Shiraishi, J. Math. Phys. 34 (4) (1993) 1480.

42. M.J. Duff and J.X. Lu, Nucl. Phys. B390 (1993) 276.

43. A.G. Felce and T.M. Samols, Phys. Lett. B308 (1993) 30.

44. Bluhm, P. Goddard and L. Dolan, Nucl. Phys. B289 (1987), 364; B309 (1988) 330.

45. J. Maharana and J.H. Schwarz, Nucl. Phys. B390 (1993) 3.

46. M. de Roo, Nucl. Phys. B255 (1985) 515.

47. J. Gauntlett, J. Harvey and J. Liu, Nucl. Phys. B409 (1993) 363; J. Gauntlett and J. Harvey, S-Duality and the spectrum of magnetic monopoles in heterotic string theory, preprint EFI-94-36.

48. R. Sorkin, Phys. Rev. Lett. 51 (1983) 87 ; D. Gross and M. Perry, Nucl. Phys. B226 (1983) 29.

49. R.R. Khuri, Phys. Lett. B259 (1991) 261.

50. R.R. Khuri, Nucl. Phys. B387 (1992) 315.

51. T. Banks, M. Dine, H. Dijkstra and W. Fischler, Phys. Lett. B 212 (1988) 45.

52. C.M. Hull, in preparation.

53. R. Nepomechie, Phys. Rev. D31 (1985) 1921.

54. A. Strominger, Nucl. Phys. B343 (1990) 167.

55. M.J. Duff and J.X. Lu, Phys. Rev. Lett. 66 (1991) 1402.

56. C.M. Hull, Nucl. Phys. B260 (1985) 182 ; L. Alvarez-Gaumé and P. Ginsparg, Commun. Math. Phys. 102 (1985) 311.

57. G.T. Horowitz and A.A. Tseytlin, I.C. preprints Imperial /TP/93-94/38, Imperial /TP/93-94/54. 
58. J. Dai, R.G. Leigh and J. Polchinski, Mod. Phys. Lett. A4 (1989) 2073; M. Dine, P. Huet and N. Seiberg, Nucl. Phys. B322 (1989) 301.

59. M. Atiyah and N. Hitchin, Phys. Lett. 107A (1985) 21; Phil. Trans. R. Soc. Lond. A315 (1985) 459; The Geometry and Dynamics of Magnetic Monopoles, Princeton University Press (1988).

60. B. Julia in Supergravity and Superspace, S.W. Hawking and M. Rocek, C.U.P. Cambridge, 1981.

61. B. Julia in Lectures in Applied Mathematics, AMS, vol. 21, 355, 1985.

62. M.J. Duff and J.X. Lu, Nucl. Phys. B347 (1990) 394.

63. R.W. Gebert and H. Nicolai, DESY-94-106, hep-th 9406175.

64. Supergravity Theories in Diverse Dimensions, A. Salam and E. Sezgin (eds.), World Scientific, Singapore.

65. A. Sen, Tata Inst. preprint TIFR-TH-94-19, hep-th 9408083.

66. E.Cremmer, in Supergravity and Superspace, S.W. Hawking and M. Rocek, C.U.P. Cambridge, 1981.

67. C. Teitelboim, Phys. Lett. B67 (1986) 63 and 69.

68. A. Achúcarro, J. Evans, P.K. Townsend and D. Wiltshire, Phys. Lett. 198B (1987) 441.

69. C.M. Hull, Phys. Lett. 139B (1984) 39.

70. M.J. Duff, G.W. Gibbons and P.K. Townsend, Phys. Lett. 332 B (1994) 321.

71. A. Sen, Phys.Lett. B329, (1994) 217.

72. C. Vafa and E. Witten, Harvard preprint HUTP-94-A017, hep-th/9408074.

73. P.K. Townsend, Phys. Lett. 202B (1988) 53. 